\title{
Lightning current and voltage distribution of large axially symmetric Buddhist stupa in Sri Lanka
}

\begin{abstract}
This is one of the first scientific investigations on lightning current and voltage distribution along Buddhist Stupa in Sri Lanka and elsewhere. Such distribution provide vital information on lightning safety concerns of people and objects outside the structure as the building is totally sealed. The metal casket of which the block of quartz is installed at the pinnacle of the Stupa, may act as the point of interception with lightning stepped leader. Large hem ispherically symmetric outer structure of the Stupa causes uniform distribution of current over its surface. Such distribution yields very low current density thus possibilities of side flashing or localized heating are minimized. Structure of the Stupa was analyzed as a collection of lumped circuit elements using MatLab and SimuLink software to show that there will be no dangerous potentials developed within possible arcing range to the surrounding, in the application of impulse current. Thinly distributed current, driven into the deeply laid foundation of the structure, prevents development of significant step potentials in the vicinity that could pose danger to the devotees.
\end{abstract}

Keyword: Lightning; Hazard prevention; Side flashing; Human safety; Step potential 